Journal of ISMAC (2020)

Vol.02/ No. 01

Pages: $38-49$

http://irojournals.com/iroismac/

DOI: https://doi.org/10.36548/jismac.2020.1.004

\title{
Improved Response Time and Energy Management for Mobile Cloud Computing Using Computational Offloading
}

\author{
Dr. Jennifer S. Raj, \\ Professor, \\ Department of ECE, Gnanamani College of Technology, \\ Namakkal, India. \\ Email: jennifer.raj@gmail.com
}

\begin{abstract}
The mobile devices capabilities are found to be greater than before by utilizing the cloud services. There are various of service rendered by the cloud paradigm and the mobile devices usually allows the execution of the resource-intensive applications on the resource- constrained mobile device to be offloaded to the cloudlets that are resource rich thus enhancing the its processing capabilities. But accessing the cloud services within the minimum response time and energy consumption still remains as a serious research problem. So the proposed method put forth in the paper scopes in developing a frame work to choose the optimal cloud service provider. The frame work proposed is categorized into two stages where the initial stage engages the classifier to segregate the mobile device according to the fuzzy K-nearest neighbor and cultivates an improved computational offloading employing the Hidden Markov Model and ACO- ant colony optimization. The algorithm proffered is implemented in the MATLAB version 9.1 and the performance is evinced on the basis of the response time, energy consumption and the processing cost. The results obtained through the proposed method proves to provide an $89 \%$ better response time, $95 \%$ better energy consumption and $50 \%$ enhanced processing cost compared to the few existing computational offloading methods put forth for the mobile cloud computing.
\end{abstract}

Keywords: Mobile Cloud Computing, Computational Offloading, Response Time, Energy Consumption, Fuzzy KNN , Hidden Markov Model and Ant colony optimization.

\section{Introduction}

The smart phones are capable of supporting multiple applications as it has a better computing competences and memory. The utilization of the applications that aid the social network and the multimedia are drastically increased, few applications automatically starts running in back ground without the knowledge of the user, resulting in enormous battery consumption causing frequently battery recharges. So the smart phone requires a most apt system that would improves its efficacy in terms of storage and the processing.

ISSN: 2582-1369 (online) 
Journal of ISMAC (2020)

Vol.02/ No. 01

Pages: $38-49$

http://irojournals.com/iroismac/

DOI: https://doi.org/10.36548/jismac.2020.1.004

In the recent days the utilization of the mobile integrated with the cloud that is termed as mobile cloud computing is becoming more and more prominent as their capabilities are advancing progressively and utilized in our daily activities. The mobile computing paradigm is comprised of the cloud computing, the wireless network and the mobile computing technologies to process the aforesaid application in a shared manner.

The cloud computing that renders service such as the platform, software and the infrastructure on demand are very advantages to the mobile devices as the cloud is highly scalable with vast storage capability. The mobile devices still faces issues, due to the limited resource capacities and the wireless connections that are unstable with the limited energy capacity and the simultaneous location changes even if it's equipped with good computing capacity. All the above mentioned makes the mobile devices to crave more for computing power and provide a better performance. "The continuous increase in the usage of the resource intensive and the power hungry mobile applications, the cloud services extended as infrastructure as service would be of very good assistance. If the applications of the mobile devices are computation intensive or less interactive, then the processing- offloading services could be utilized. Subsequently there is cloud service could afford multiple computations." so it is always important to select the services based on the requirements of the cloud and it is also essential to note whether the service provider is closer by in order to reduce the response time, the energy consumption and the cost of the computation. To bring down the latencies observed in receiving the responses the cloudlets were preferred, the cloudlets are often termed as the components found at the edge. It was found from the literature survey that the offloading of application to be computed through the cloud modules that are edge based proved to have a decreased processing time in a cost effective manner.

So the proposed method in the paper puts forth the system model for the offloading the computationally intensive mobile applications. By categorizing the mobile devices based on their distance to the particular cloud services using the Fuzzy KNN framework and utilizing the Hidden Markov Model to enhance the performance of the offloading, the paper is arranged with the related works in 2, the proposed work providing the particulars of the computational offloading services, the criteria for selecting the services of the cloudlet, and the improving the service employing HMM are elaborated in 3 . The analysis of the performance is done in 4 , and the Conclusion in 5.

\section{Related Works}

ISSN: 2582-1369 (online) 
Journal of ISMAC (2020)

Vol.02/ No. 01

Pages: $38-49$

http://irojournals.com/iroismac/

DOI: https://doi.org/10.36548/jismac.2020.1.004

Wu et al [1] . proposes the "Optimal cloud-path selection in mobile cloud offloading systems based on QoS criteria." Chen et al [2] describes the "Efficient multi-user computation offloading for mobile-edge cloud computing." Bhalaji, N. et al [3] puts forth an "Efficient and Secure Data Utilization in Mobile Edge Computing by Data Replication." Where the author Zheng et al [4] elaborates the "stochastic gametheoretic approach for the Dynamic computation offloading for mobile cloud computing" Kumar et al [5] discusses the "Review on task scheduling in ubiquitous clouds." put forth the answers for the hypothesis such the challenges and issues related to the scheduling. ur Rehman, et al [6] discusses the approach for the selection of cloud service based on the multiple criteria's

Bhalaji, $\mathrm{N}$ et al [7] puts forth the strategies for the "delay diminished efficient task scheduling and allocation for heterogeneous cloud environment." and Pandian, M. Durai. et al 8] presents the enhanced network performance and mobility management of IoT multi networks." whereas the Bestak et al [9] discusses the strategies for "big data analytics for smart cloud-fog based applications." and the author Shakya, Subarna et al [10] proposes the data migration in the cloud with the efficient security structure"

Karunakaran, V. et al [11] puts forth the task scheduling algorithm for the cloud paradigm minimizing the cost and the execution time. Bashar, et al [12] puts forth the. "Secure and cost efficient implementation of the mobile computing using offloading technique." and the author Kumar et al [13] present the "A novel report on architecture, protocols and applications in Internet of Things (IoT)"

The "Cloudlet dynamic server selection policy for mobile task off-loading in mobile cloud computing using soft computing techniques" was put forth by the Rashidi, et al [14] to reduce the burden over the cloud. And the "Computation offloading for service workflow in mobile cloud computing" was put forth by the Deng et al [15] to enhance the computational capabilities of the cloud. Kim, et al [16] presents the tutorial of "Fuzzy K-NN algorithm" Resch, et al [17] and Katiyar, et al [18] presents the tutorial of the HMM and ACO respectively.

\section{Proposed Work}

ISSN: 2582-1369 (online) 
Journal of ISMAC (2020)

Vol.02/ No. 01

Pages: $38-49$

http://irojournals.com/iroismac/

DOI: https://doi.org/10.36548/jismac.2020.1.004

The increasing number of mobile users and increasing demands to accesses the service ubiquitously irrespective of time and place, and has led to the necessity of the cloud integrated mobile devices. This actually enhances the processing and the storage capacities of the mobile devices. Despite the cloud capabilities the serious problem faced in the mobile device integrated with the cloud computing is the increased energy consumption, response time and the cost of the computation as the proper method to select the perfect cloudlet is still questionable. So the proposed method puts forth the method to have a perfect decision making by considering the device mobility and enables the device to access the edge device or the cloud whichever is closer to it. For this, the method utilizes the FUZZY k-nearest neighbor to categorizes the devices based it range and to allot the perfect cloud services to the mobile devices and utilizes to HMM in identifying the probable trails between the resource at the edge or the cloud and the mobile-devices

\subsection{Fuzzy K-Nearest Neighbor in Resources Selection}

The apt resource at the edge for the mobile device is carefully chosen employing the K-nearest neighbor classifier and the fuzzy rules [16]. The K-nearest neighbor that distinguishes the membership between the classified and the unclassified data set of the labeled class is utilized in the proposed method to categorize the range of the mobile device and equips them identify the resource at the edge or the cloud that provides the requested service.

The algorithm below in fig. 1 describes the intervention of the fuzzy and the K-nearest neighbor in allotting the perfect resource to the mobile devices based on their request.

ISSN: 2582-1369 (online) 
Journal of ISMAC (2020)

Vol.02/ No. 01

Pages: $38-49$

http://irojournals.com/iroismac/

DOI: https://doi.org/10.36548/jismac.2020.1.004

Input : mobile Device requisition (MDR), Edge Resources $(E R)$,

Cloud Services (CS)

Output : Allot service to Mobile users

Begin

Receive $M D R$

For all $M D R$

Begin

Determine the distance between the device and the edge resources

Determine the range of access

Estimate the $E R$ at proximity

Apply K-nearest Neighbor

Estimate Minkowski distance $=\left(\sum_{i=1}^{n}\left|x_{i}-y_{i}\right|^{p}\right)^{\frac{1}{p}}$

If distance is less than expected

Add the $E R_{1}$ to K-nearest neighbor set

Else

Repeat distance estimation

Stop

If nearest $E R$ is found

Else

Assign the MDR to CS

Stop

Stop

Figure.1Fuzzy K-Nearest Neighbor Based Service Allotment

\subsection{Path prediction based on the HMM and ACO}

As the devices used are mobile in nature, it becomes essential to estimate the mobility of the device to determine the proper trail between the mobile-devices and the resource at the edge or the cloud. So the proposed method utilizes the hidden-markov-model [17] that hides the states unlike the markov model and displays only the output states to predict the probable trails considering the previous trails of the mobile

ISSN: 2582-1369 (online) 
Journal of ISMAC (2020)

Vol.02/ No. 01

Pages: $38-49$

http://irojournals.com/iroismac/

DOI: https://doi.org/10.36548/jismac.2020.1.004

device, The prediction procedure in the hidden-markov-model takes two strides, the first step the optimal parameters are determined using the parameter learning and in the second step estimates the interference that was acquired in the prediction. The steps involved in the prediction of the devices are as follows.

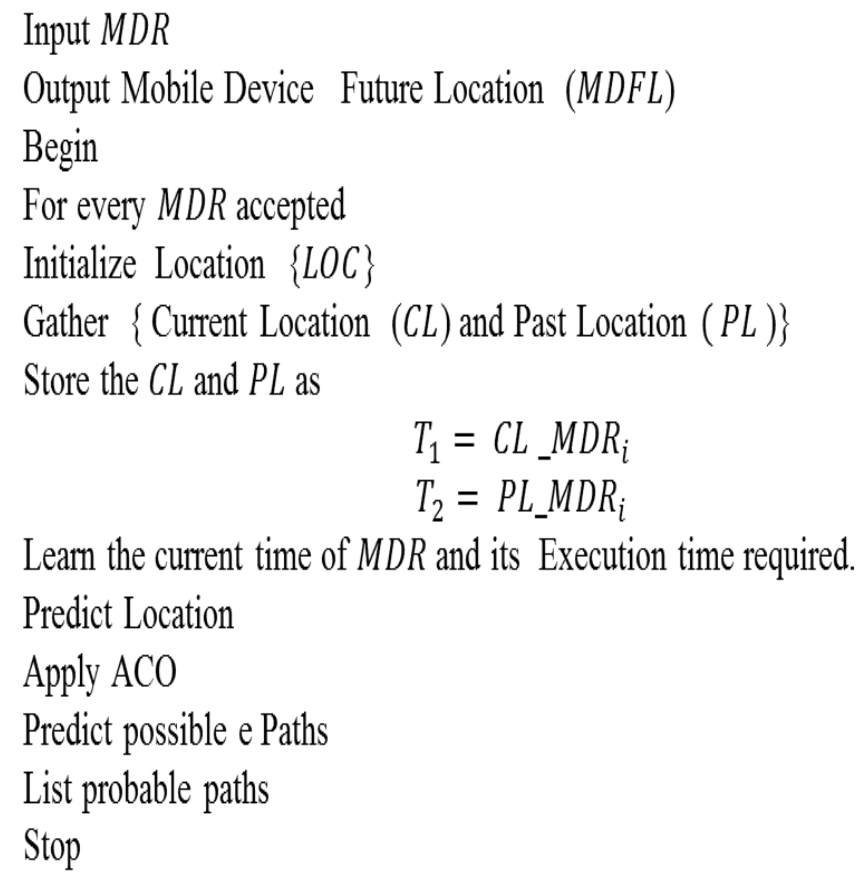

Figure .2 Algorithm to Identify the Probable Path Using the HMM and ACO

\subsection{Proposed Improved Computational Offloading}

The mobile devices that request for the computational offloading to the cloud are initially segregated based on their ranges and identified with the proper and nearest resources using the fuzzy K- nearest neighbor. Once the resources are allotted now the probable paths are identified, the motility of the each devices are acquired here the mobility of the devices cause the devices to change its location and breaking the connection established from the cloud or the cloudlet. So the proposed method utilizes the HMM to predicts the future location of the mobile device based on its movement in the past. The prediction of the mobile devices location is followed by the probable path identification with the devices and cloud using the ACO

ISSN: 2582-1369 (online) 
Journal of ISMAC (2020)

Vol.02/ No. 01

Pages: $38-49$

http://irojournals.com/iroismac/

DOI: https://doi.org/10.36548/jismac.2020.1.004

(Ant colony optimization) [18] - that identifies that shortest path for its food utilizing its pheromone update. The block diagram below provides the over view of the proposed computation offloading.

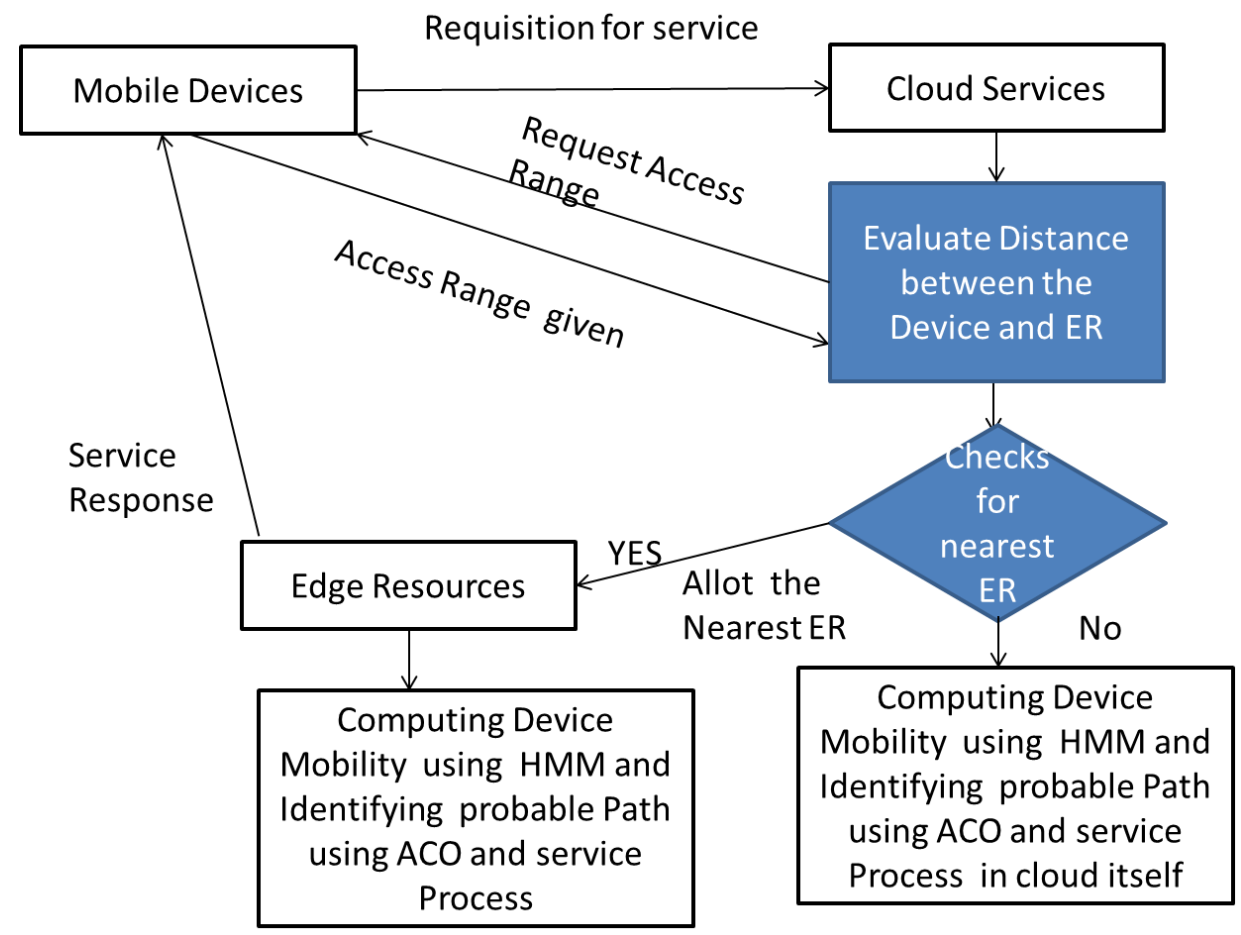

Figure .3 Dynamic Offloading Process

The proposed method with the perfect resource selection utilizing the Fuzzy rule based K-nearest neighbor followed by the estimation of the mobile devices location and identifying the shortest path between the mobile-devices and the resources either in the edge or the cloud utilizing the hidden-markovmodel and the ACO respectively outstrips the existing methodologies and proves to be conclusive.

\section{Performance Analysis}

The proposed method is validated using the network simulator-2 with the hardware configurations such as Intel core i9 ,16 Gb RAM, 2 Tb HDD and 128 GB SSD. With the fusion of resource at edge for processing the services, the proposed method is evaluated on the basis of number mobile user who request for the cloud

ISSN: 2582-1369 (online) 
Journal of ISMAC (2020)

Vol.02/ No. 01

Pages: $38-49$

http://irojournals.com/iroismac/

DOI: https://doi.org/10.36548/jismac.2020.1.004

services to evince the capacity of the method on terms of the energy consumption, response time and cost of computation. The table. 1 below provides the simulation parameters.

\begin{tabular}{|l|l|}
\hline \multicolumn{1}{|c|}{ Parameter } & \multicolumn{1}{c|}{ Values } \\
\hline Number of Mobile Users & $50-100$ \\
\hline Simulation area & $200 \mathrm{~m}$ \\
\hline Total Power of Transmission & $100 \mathrm{Mw}$ \\
\hline Channel Bandwidth & $2 \mathrm{Mhz}$ \\
\hline Processing Speed of ER & 1100 Mega Cycles \\
\hline Processing Speed of CS & 1300 Mega Cycles \\
\hline
\end{tabular}

Table .1 Simulation Parameters

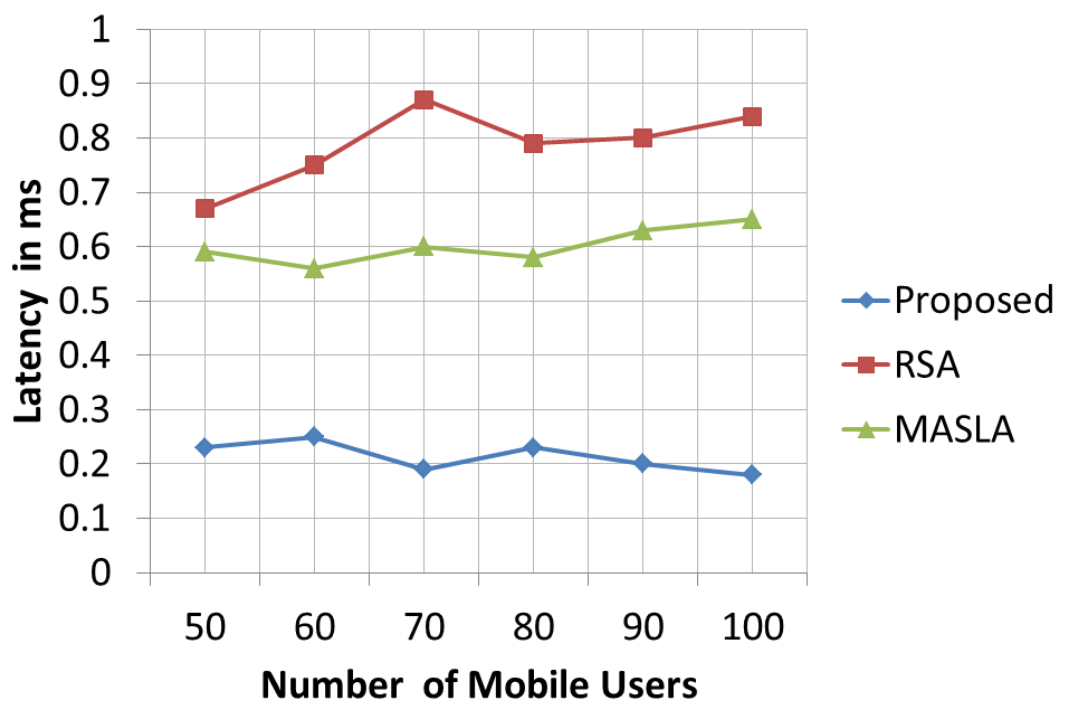

ISSN: 2582-1369 (online) 
Journal of ISMAC (2020)

Vol.02/ No. 01

Pages: $38-49$

http://irojournals.com/iroismac/

DOI: https://doi.org/10.36548/jismac.2020.1.004

The figure. 4 above represents the results observed on the energy consumption of the proposed method, the results obtained shows that the proposed method is $95 \%$ better energy consumption than the existing approaches such as random selection algorithm and multi agent stochastic learning algorithm.

The Figure. 5 below shows the response time of the proposed method; the results obtained shows that the proposed method is $89 \%$ better than the existing approaches such as RSA-random selection algorithm and MASLA- multi agent stochastic learning algorithm. But still faces difficulties and the increased response time once if prediction goes wrong or the mobile devices moves beyond the vicinity of the services already fixed. This would cause more time as the procedure starts from the first step, initiating the identification of the service, then the location and the path

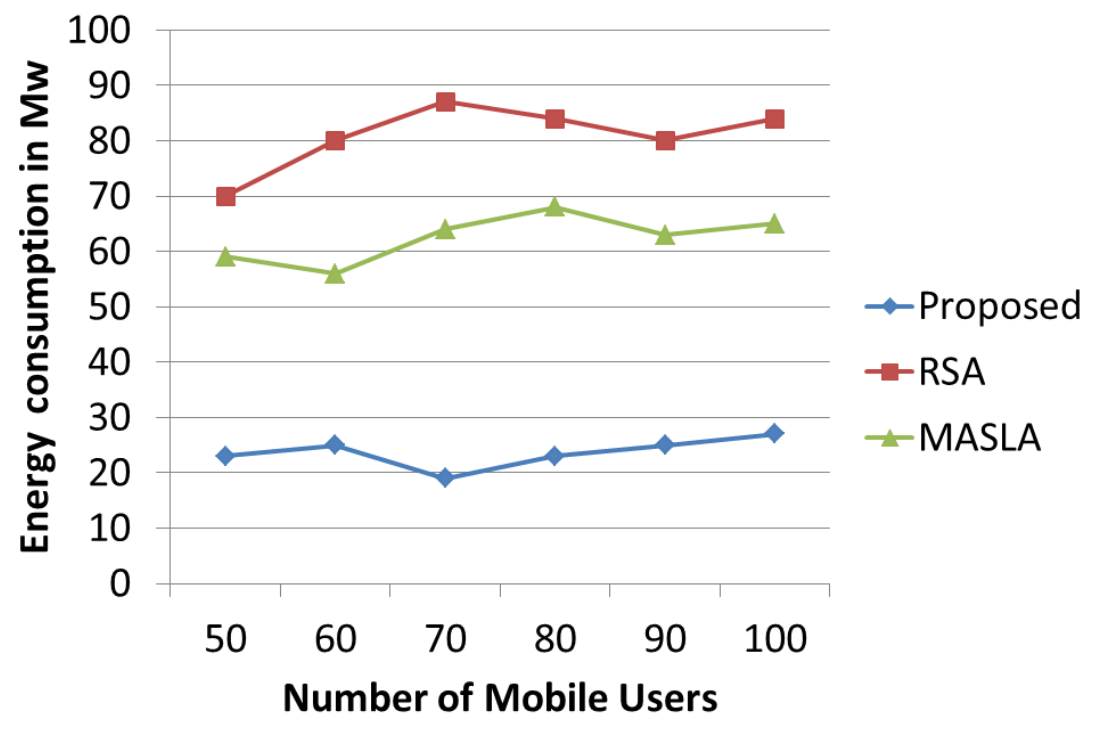

The table. 2 below is the results of the cost that was spent on the computation, the results details the particulars of the cost consumed by the various number of clients and the results obtained shows the percentage of cost consumed by the approach proposed and the other methods such as the random selection algorithm and multi agent stochastic learning algorithm, this shows the proposed method has a better 50\% less cost computation compared to the random selection algorithm and multi agent stochastic learning algorithm.

ISSN: 2582-1369 (online) 
Journal of ISMAC (2020)

Vol.02/ No. 01

Pages: $38-49$

http://irojournals.com/iroismac/

DOI: https://doi.org/10.36548/jismac.2020.1.004

\begin{tabular}{|c|c|c|c|}
\hline $\begin{array}{c}\text { Number of } \\
\text { users }\end{array}$ & $\begin{array}{c}\text { Random } \\
\text { selection } \\
\text { Algorithm }\end{array}$ & $\begin{array}{c}\text { Multi Agent } \\
\text { Stochastic } \\
\text { Learning } \\
\text { Algorithm }\end{array}$ & Proposed \\
\hline 50 & 188.126 & 53.09 & 20.34 \\
\hline 60 & 300.675 & 76.78 & 24.35 \\
\hline 70 & 576.918 & 87.56 & 36.34 \\
\hline 80 & 785.03 & 93.45 & 48.56 \\
\hline 90 & 823.56 & 110.23 & 67.45 \\
\hline 100 & 1023.45 & 150.45 & 70.38 \\
\hline
\end{tabular}

Table. 2 Cost Computation.

Where the cost for each computation is estimated by adding the energy $\left(C_{e}\right)$ and time $\left(C_{t}\right)$ required for processing multiplied by the size of the data, $\left(D_{s}\right)$ for the number of mobile users $\left(M_{u}\right)$, this is further added to the product of the total time consume for the number of the mobile users requested as stated in equation 1

$$
\text { Computation }_{\text {Cost }}=\frac{\left(C_{e}+C_{t}\right) D_{s}}{M_{u}}+\left(\frac{C_{t} D_{s}}{M_{u}}\right)
$$

\section{Conclusion}

The proposed method of the paper has put forth a computational offloading strategy so as to bring down the response time latencies, the energy consumption and the cost of the computation by employing the fuzzy K-Nearest Neighbor, the hidden markov model to identify the proper services and envisage the future location of the mobile devices based on its past locations respectively and employs ant colony optimization for the purpose of identifying the shortest path with the between the resources allotted and the mobile devices. The results obtained from the validation of the proffered method with the network simulator-II shows that the approach put forth shows considerable improvement in the latencies, cost and the energy consumption compared to the conventional methodologies such as the RSA and the MASLA.

ISSN: 2582-1369 (online) 
Journal of ISMAC (2020)

Vol.02/ No. 01

Pages: $38-49$

http://irojournals.com/iroismac/

DOI: https://doi.org/10.36548/jismac.2020.1.004

\section{References}

[1] Wu, Huaming, Qiushi Wang, and Katinka Wolter. "Optimal cloud-path selection in mobile cloud offloading systems based on QoS criteria." International Journal of Grid and High Performance Computing (IJGHPC) 5, no. 4 (2013): 30-47.

[2] Chen, $\mathrm{Xu}$, Lei Jiao, Wenzhong $\mathrm{Li}$, and Xiaoming Fu. "Efficient multi-user computation offloading for mobile-edge cloud computing." IEEE/ACM Transactions on Networking 24, no. 5 (2015): 2795-2808.

[3] Bhalaji, N. "Efficient and Secure Data Utilization in Mobile Edge Computing By Data Replication." Journal of ISMAC 2, no. 01 (2020): 205-216.

[4] Zheng, Jianchao, Yueming Cai, Yuan Wu, and Xuemin Shen. "Dynamic computation offloading for mobile cloud computing: A stochastic game-theoretic approach." IEEE Transactions on Mobile Computing 18, no. 4 (2018): 771-786.

[5] Kumar, Dinesh. "Review on task scheduling in ubiquitous clouds." J. ISMAC 1, no. 01 (2019): 72-80.

[6] ur Rehman, Zia, Farookh K. Hussain, and Omar K. Hussain. "Towards multi-criteria cloud service selection." In 2011 Fifth International Conference on Innovative Mobile and Internet Services in Ubiquitous Computing, pp. 44-48. Ieee, 2011.

[7] Bhalaji, N. "delay diminished efficient task scheduling and allocation for heterogeneous cloud environment." Journal of trends in Computer Science and Smart technology (TCSST) 1, no. 01 (2019): 51-62.

[8] Pandian, M. Durai. "enhanced network performance and mobility management of iot multi networks." Journal of trends in Computer Science and Smart technology (TCSST) 1, no. 02 (2019): 95-105.

[9] Bestak, Robert, and S. Smys. "big data analytics for smart cloud-fog based applications." Journal of trends in Computer Science and Smart technology (TCSST) 1, no. 02 (2019): 74-83.

[10] Shakya, Subarna. "an efficient security framework for data migration in a cloud computing environment." Journal of Artificial Intelligence 1, no. 01 (2019): 45-53.

[11] Karunakaran, V. "a stochastic development of cloud computing based task scheduling algorithm." Journal of Soft Computing Paradigm (JSCP) 1, no. 01 (2019): 41-48.

[12] Bashar, Abul. "Secure and cost efficient implementation of the mobile computing using offloading technique." Journal of Information Technology 1, no. 01 (2019): 48-57.

[13] Kumar, R. Praveen, and S. Smys. "A novel report on architecture, protocols and applications in Internet of Things (IoT)." In 2018 2nd International Conference on Inventive Systems and control (ICISC), pp. 1156-1161. IEEE, 2018.

ISSN: 2582-1369 (online) 
Journal of ISMAC (2020)

Vol.02/ No. 01

Pages: $38-49$

http://irojournals.com/iroismac/

DOI: https://doi.org/10.36548/jismac.2020.1.004

[14] Rashidi, Shima, and Saeed Sharifian. "Cloudlet dynamic server selection policy for mobile task off-loading in mobile cloud computing using soft computing techniques." The Journal of Supercomputing 73, no. 9 (2017): 3796-3820.

[15] Deng, Shuiguang, Longtao Huang, Javid Taheri, and Albert Y. Zomaya. "Computation offloading for service workflow in mobile cloud computing." IEEE transactions on parallel and distributed systems 26, no. 12 (2014): 3317-3329.

[16] Kim, Yooii K., and Joon H. Han. "Fuzzy K-NN algorithm using modified K-selection." In Proceedings of 1995 IEEE International Conference on Fuzzy Systems., vol. 3, pp. 16731680. IEEE, 1995.

[17] Resch, Barbara. "Hidden Markov Models A Tutorial for the Course Computational Intelligence." (2004).

[18] Katiyar, Sapna, N. Ibraheem, and Abdul Quaiyum Ansari. "Ant colony optimization: a tutorial review." MR Int. J. Eng. Technol 7, no. 2 (2015): 35-41.

ISSN: 2582-1369 (online) 